\title{
Characterisation and heterologous biosynthesis of burnettiene $A$, a new polyene- decalin polyketide from Aspergillus burnettii
}

\author{
Indra Roux, a Simon Bowles, b John A. Kalaitzis, ${ }^{b, c}$ Daniel Vuong, ${ }^{b}$ Ernest Lacey, ${ }^{b, c}$ Yit-Heng Chooi ${ }^{\text {a* }}$ and Andrew M. Piggott ${ }^{c *}$ \\ a School of Molecular Sciences, The University of Western Australia, Perth, WA 6009, Australia. E-mail: yitheng.chooi@uwa.edu.au \\ b Microbial Screening Technologies Pty. Ltd., Smithfield, NSW 2164, Australia \\ cDepartment of Molecular Sciences, Macquarie University, Sydney, NSW 2109, Australia. E-mail: andrew.piggott@mq.edu.au
}

\begin{abstract}
Chemical exploration of the recently described Australian fungus, Aspergillus burnettii, uncovered a new metabolite, burnettiene A. Here, we characterise the structure of burnettiene $A$ as a polyene-decalin polyketide. Bioinformatic analysis of the genome of $A$. burnettii identified a putative biosynthetic gene cluster for burnettiene A (bue), consisting of eight genes and sharing similarity to the fusarielin gene cluster. Introduction of the reassembled bue gene cluster into Aspergillus nidulans for heterologous expression resulted in the production of burnettiene $A$ under native promoters. Omission of bueE encoding a cytochrome P450 led to the production of preburnettiene A, confirming that BueE is responsible for catalysing the regiospecific multi-oxidation of terminal methyl groups to carboxylic acids. Similarly, bueF was shown to encode an ester-forming methyltransferase, with its omission resulting in the production of the tricarboxylic acid, preburnettiene B. Introduction of an additional copy of the transcription factor bueR under the regulation of the gpdA promoter significantly improved the heterologous production of the burnettienes. Burnettiene A displayed strong in vitro cytotoxicity against mouse myeloma NS-1 cells (MIC 0.8 $\mu \mathrm{g} / \mathrm{mL})$.
\end{abstract}

\section{Introduction}

Filamentous fungi from the genus Aspergillus are prolific producers of secondary metabolites which display a diverse range of bioactivities. ${ }^{1}$ As part of our ongoing chemotaxonomic exploration of rare and talented Australian Aspergilli, ${ }^{3-10}$ we recently reported the novel species Aspergillus burnettii, isolated from soil collected in Queensland, Australia. ${ }^{4}$ Chemical profiling of $A$. burnettii revealed a variety of unprecedented secondary metabolites, including the polyketide-peptide hybrid burnettramic acids ${ }^{6}$ which displayed potent antifungal activity. Continuing the chemical exploration of $A$. burnettii, herein we report the structure, biological activity and biosynthesis of the novel polyene-decalin polyketide, burnettiene A (1).

Secondary metabolites containing a decalin motif present high structural and functional diversity due to the multiple possible substitutions on the decalin scaffold and its pendent chains. ${ }^{11}$ Several fungal decalin polyketides present remarkable biological activities (Fig. 1). For example, lovastatin is widely used as a cholesterol-lowering agent, while equisetin shows HIV integrase inhibitory activity. ${ }^{12,13}$ Fusarielin $A$, isolated from various Fusarium species, presents antifungal, antiangiogenic and antiproliferative activities. ${ }^{14-17}$ Other fungal decalins include betaenones $A-C$, which show phytotoxic activity, and campafungins $A-D$, which display promising activity against Cryptococcus neoformans. ${ }^{18,19}$

Bioinformatic analysis of our recently published $A$. burnettii genome sequence ${ }^{4}$ has also allowed us to identify the putative biosynthetic gene cluster (BGC) responsible for the biosynthesis of 1 (bue) and to explore synthetic biology approaches for the production of $\mathbf{1}$, given that $A$. burnettii only produces modest amounts of $\mathbf{1}$ amongst a suite of other major cometabolites. Heterologous expression of the bue cluster in Aspergillus nidulans with transcription factor overexpression has provided valuable insights into the biosynthesis of $\mathbf{1}$ and has provided an alternative source to access 1 and its pathway intermediates for bioactivity studies.

\section{Results and discussion}

Burnettiene A from Aspergillus burnettii is a polyketide-derived decalin with an unusually long polyene side chain

Burnettiene A (1) was previously isolated from A. burnettii MST FP2249 cultivated on pearl barley. ${ }^{4}$ HRESI(-)MS analysis of 1 revealed a deprotonated molecule $\left[\mathrm{M}-\mathrm{H}^{-} \mathrm{m} / \mathrm{z} 537.2860\right.$ indicative of a molecular formula $\mathrm{C}_{32} \mathrm{H}_{42} \mathrm{O}_{7} \quad(\Delta \mathrm{mmu} \quad 0.2)$ requiring 12 double bond equivalents (DBE) (Fig. S $8^{+}$). The UVvis spectrum of $\mathbf{1}$ (Fig. $\mathrm{S7+}$ ) shows a distinctive series of maxima at $308(33 \%), 318$ (63\%), 334 (100\%) and 354 (96\%) nm, characteristic of an isolated pentaene system. Examination of the ${ }^{13} \mathrm{C}$ NMR spectrum of $\mathbf{1}$ (Fig. $\mathrm{S} 2+$ ) revealed resonances attributable to 3 carboxylic acid/ester carbonyl carbons ( $\delta c$ $166.3,167.1,175.8), 14$ olefinic carbons ( $\delta_{c} 118.7$ to 159.6$), 2$ oxygenated carbons ( $\delta_{\mathrm{C}} 51.7$ and 72.9 ) and 13 aliphatic carbons ( $\delta_{\mathrm{c}} 12.8$ to 56.2 ), thus accounting for 10 of the $12 \mathrm{DBE}$ and requiring 1 to be bicyclic. The ${ }^{1} \mathrm{H}$ NMR spectrum of 1 (Fig. S1+) confirmed the presence of an unsubstituted pentaene chain, with 8 overlapping resonances between $\delta_{H} 6.17-6.36(\mathrm{H}-15$ to $\mathrm{H}-22)$ and 2 isolated resonances at $\delta_{\mathrm{H}} 5.31(\mathrm{H}-14 ; \mathrm{d}, J=15.5 \mathrm{~Hz})$ and $\delta_{\mathrm{H}} 5.65(\mathrm{H}-23 ; \mathrm{dd}, J=14.2,7.0 \mathrm{~Hz})$ delineating the pentaene system. The ${ }^{1} \mathrm{H}-{ }^{1} \mathrm{H}$ COSY spectrum of 1 (Fig. S5 ${ }^{+}$) revealed the presence of a spin system extending beyond $\mathrm{H}-23$, incorporating a hydroxymethine $\left(\mathrm{H}-24 ; \delta_{\mathrm{H}} 4.13, \mathrm{dd}, J=7.2,7.0\right.$ $\mathrm{Hz})$ and a methylmethine $\left(\mathrm{H}-25 ; \delta_{\mathrm{H}} 2.35, \mathrm{dq}, J=7.2,7.2 \mathrm{~Hz}\right.$ and 25-Me; $\delta_{\mathrm{H}} 0.91, \mathrm{~d} J=7.2 \mathrm{~Hz}$ ) (Fig. 2). The ${ }^{1} \mathrm{H}-{ }^{13} \mathrm{C} \mathrm{HMBC}$ spectrum of 1 (Fig. S4t) shows correlations from H-24, $\mathrm{H}-25$ and $25-\mathrm{Me}$ to C-26 ( $\left.\delta_{\mathrm{c}} 175.8\right)$ (Fig. 2 and Fig. S4 ${ }^{\dagger}$ ), indicating the chain must be terminated by a carboxylic acid. Further analysis of the COSY spectrum revealed a second spin system extending from $\mathrm{H}-6$ 
<smiles>COC(=O)C1=C[C@H]2C[C@H](C)CC[C@H]2[C@H](/C=C/C=C/C=C/C=C/C=C/[C@@H](O)[C@@H](C)C(=O)O)[C@H]1/C=C(\C)C(=O)O</smiles><smiles>C/C=C(\C)[C@]1(O)C=C[C@H]2CC(C)=CC[C@H]2[C@H]1/C=C/C=C/C=C/C=C/C(=O)O</smiles>

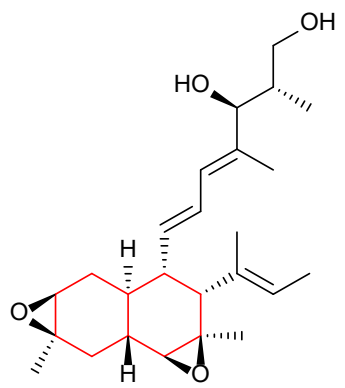

Fusarielin A

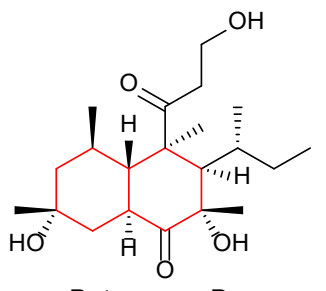

Betaenone B<smiles>CC[C@H](C)C(=O)O[C@H]1C[C@H](C)C=C2C=C[C@H](C)[C@H](CC[C@@H]3C[C@@H](O)CC(=O)O3)[C@H]21</smiles>

Lovastatin<smiles>C/C=C/[C@H]1C=C[C@H]2C[C@@H](C)CC[C@H]2[C@]1(C)/C(O)=C1/C(=O)C(CO)N(C)C1=O</smiles>

Equisetin

Figure 1 Representative fungal natural products containing a decalin motif (decalin highlighted in red), including burnettiene A (1) reported in this study.

to $\mathrm{H}-12$, including 9-Me, with a diagnostic COSY correlation between $\mathrm{H}-7$ and $\mathrm{H}-12$ indicating the presence of a cyclohexyl ring. $\mathrm{HMBC}$ correlations from $\mathrm{H}-14$ to $\mathrm{C}-12, \mathrm{C}-13$ and $13-\mathrm{Me}$, and from $\mathrm{H}-12$ to $\mathrm{C}-13,13-\mathrm{Me}$ and $\mathrm{C}-14$ confirmed $\mathrm{C}-13$ as the shared point of attachment of the cyclohexyl ring and the pentaene-containing chain. Additional $\mathrm{HMBC}$ correlations from $\mathrm{H}-4$ to $\mathrm{C}-5, \mathrm{C}-6, \mathrm{C}-12, \mathrm{C}-13$ and $13-\mathrm{Me}$, from H-6 to $\mathrm{C}-4, \mathrm{C}-5$ and $\mathrm{C}-12$, and from $\mathrm{H}-14$ to $\mathrm{C}-4$ defined a decalin ring system fused at C-7 and C-12, and containing a $\Delta^{5,6}$ double bond. Next, HMBC correlations from $\mathrm{H}-4, \mathrm{H}-6$ and a putative methoxy group at $\delta_{\mathrm{H}}$ $3.59(3 \mathrm{H}, \mathrm{s})$ to $\delta_{\mathrm{C}} 166.3$ positioned a carboxylic acid methyl ester at $\mathrm{C}-5$. Finally, $\mathrm{HMBC}$ correlations from $\mathrm{H}-2$ and 3-Me to $\mathrm{C}-4$ and from $\mathrm{H}-2$ to $\mathrm{C}-1\left(\delta_{\mathrm{C}} 167.1\right)$ confirmed the presence of a 3substituted but-2-enoic acid side chain attached at C-4. The relative configuration of the trans-decalin system was established by detailed analysis of the ${ }^{1} \mathrm{H}-{ }^{1} \mathrm{H}$ ROESY spectrum of 1 (Fig. S6+), with diagnostic correlations between $\mathrm{H}-4, \mathrm{H}-7, \mathrm{H}-9$, $\mathrm{H}-10 \mathrm{a}, \mathrm{H}-11 \mathrm{~b}$ and $13-\mathrm{Me}$ on the top face, and between $\mathrm{H}-10 \mathrm{~b}$ and $\mathrm{H}-12$ on the bottom face (Fig. 2). Unfortunately, attempts at crystallising $\mathbf{1}$ to determine the absolute configuration of the molecule were unsuccessful. However, we have tentatively assigned a $24 R, 25 R$ configuration based on detailed genomic analysis (discussed below).

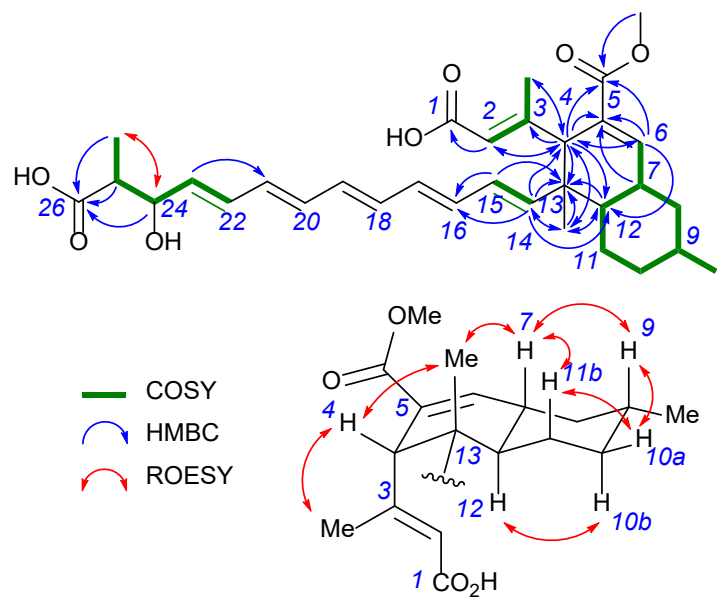

Figure 2 Selected 2D NMR correlations for burnettiene A (1)

Comparative genomic analysis uncovers putative bue gene cluster and homologues in other species

In fungal secondary metabolites, the decalin scaffold is typically synthesised by an iterative type I highly reducing polyketide synthase (HR-PKS) or by a hybrid PKS-nonribosomal peptide synthetase (PKS-NRPS). ${ }^{21}$ For example, fusarielin $A$ is synthesised from short-chain carboxylic acids by an HR-PKS, while equisetin is produced by a PKS-NRPS that additionally incorporates L-serine, forming a tetramic acid appended to a decalin in the structure (Fig. 1). ${ }^{22}$ The cyclisation from the linear polyketide substrate can occur via a Diels-Alder reaction catalysed within the PKS, or by a Diels-Alderase enzyme. ${ }^{23,24}$

Given that the genome of $A$. burnettii MST FP2249 has been sequenced, ${ }^{4}$ we attempted to identify the gene cluster encoding burnettiene $A(\mathbf{1})$. Previous genome analysis revealed that $A$. burnettii harbours 25 putative BGCs containing an HRPKS. ${ }^{4}$ Among them, the putative burnettiene gene cluster (bue) was identified by its similarity to the fusarielin cluster from Fusarium graminearum $\mathrm{PH}-1$ (Fig. 3a). ${ }^{16}$ As fusarielin is a decalin polyketide resembling $\mathbf{1}$, the bue gene cluster was selected as a candidate for the biosynthesis of $\mathbf{1}$. The HR-PKS enzyme BueA shows $50 \%$ identity with FSL1 and shared domain architecture (Fig. 3b, Table $\left.\mathrm{S}^{+}\right)$). The bue cluster also contains the transacting enoyl reductase (ER) BueB, which displays $60 \%$ identity with the trans-ER FSL5. There is also homology between the gene products BueC/D/E and FSL2/3/4 (ranging from 39-48\% identity), which are an $\alpha / \beta$ hydrolase, epimerase, and cytochrome P450, respectively (Fig. 3, Table S4+). Additionally, bueR encodes a transcription factor (TF) that presents homology to the cluster specific TF FSL7. BueR also contains a $\mathrm{Zn}_{2} \mathrm{Cys}_{6}$ DNA-binding domain, which is the most common type of cluster-specific TF. ${ }^{25}$ To define the bue gene cluster boundaries, we searched for homologues of the putative bue and neighbouring genes in NCBI GenBank with cblaster. ${ }^{26}$ We found a wide distribution of this BGC family across several fungi from the Eurotiomycetes and Sordariomycetes classes, as it was 
Fusarium graminearum $\mathrm{PH}-1$ NC_026474:1-23037 (FSL cluster)

Aspergillus burnettii MST FP2249 SPNV01000211.1:43974-65572 (bue cluster)

Aspergillus niger strain FDAARGOS_311 NKJJ02000001:2154-25122

Aspergillus clavatus NRRL 1 NW_001517095:2729-21192

Aspergillus fumigatus Af293 NC_007194:1834-17843 (Cluster 4)

b

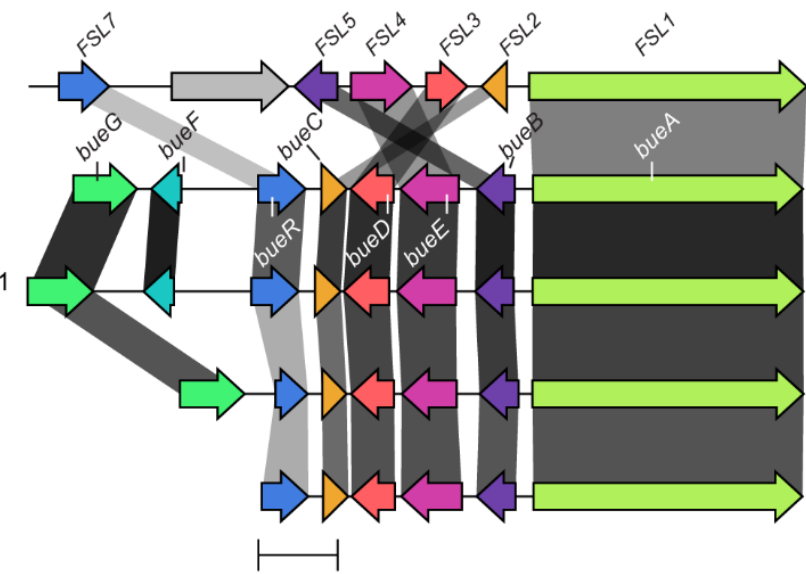

$2.5 \mathrm{~kb}$
HR-PKS

Enoylreductase

Hydrolase

Epimerase

- Cytochrome P450

Methyltransferase

- Transcription factor

- Transporter

Unknown

BueA, 2585 aa, KS-AT-DH-MT-KR-ACP

HR-PKS
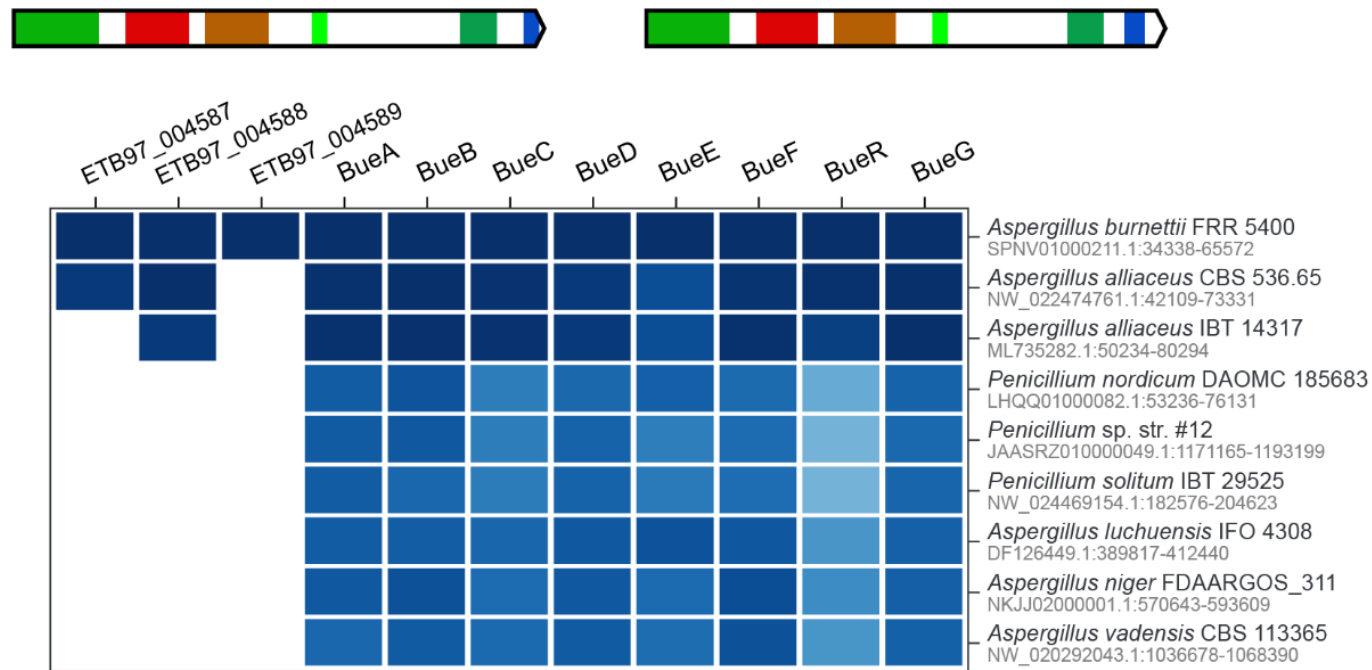

Figure 3 Bioinformatic identification of the bue gene cluster. a. Biosynthetic gene clusters homologous to the bue cluster in other fungi. Gene names from the bue cluster and fusarielin cluster (FSL) are indicated. Grayscale bars linking genes indicate amino acid identity of the encoded protein (0\% fully transparent, $100 \%$ black). Genomic coordinates are written in grey. b. HR-PKS domain architecture of FSL1 and BueA. KS, ketosynthase; AT, malonyl-CoA acyl transferase; $\mathrm{DH}$, dehydratase; $\mathrm{MT}$, methyltransferase; $\mathrm{KR}$, ketoreductase; $\mathrm{ACP}$, acyl carrier protein. $\mathrm{c}$. Representative homologous clusters with high conservation ( $\geq 48 \%$ identity across all genes) in other fungal species identified with cblaster ${ }^{26}$ in NCBI GenBank. Flanking genes that are not found colocalised in other species indicate a gene cluster boundary. A. burnettii MST FP2249 and A. burnettii FRR 5400 are isotypes.

previously observed in phylogenetic analysis of the FSL cluster. $^{27}$ Importantly, we found close similarity to uncharacterised BGCs from several Aspergilli, including A. niger, A. fumigatus and A. clavatus (Fig. 3a). For example, six gene products from the bue cluster share $74-88 \%$ identity to the homologous cluster from the opportunistic human pathogen $A$. fumigatus Af293 (Fig. 3a). This cluster, A. fumigatus cluster 4, has been extensively analysed across $A$. fumigatus strains at a genetic level by Lind and co-workers, and it was observed to undergo pseudogenisation in many strains. ${ }^{27}$ Lind et al. also attempted to find the evolutionary origin of this cluster family, but as there are many gain and loss events in both Sordariomycetes and Eurotiomycetes fungi, it remains uncertain. Interestingly, we observed that the putative methyltransferase BueF and a major facilitator superfamily (MFS) transporter BueG, which lacked homologues within the fusarielin cluster, seem to be highly conserved in other species (Fig. 3c). The flanking genes downstream of bueA were not conserved in other species, indicating a cluster boundary (Fig. 3c). As the bue cluster is located at the end of an A. burnettii genome scaffold, we decided to delimit the putative bue cluster to the genes bueA/B/C/D/E/F/G/R (ETB97_004590-004597), that are encoded within a $\sim 22 \mathrm{~kb}$ region of $A$. burnettii genome.

\section{Heterologous expression in Aspergillus nidulans strains provides} insights into burnettiene biosynthesis and gene cluster regulation

To investigate whether the putative bue cluster contains all the genes required for the biosynthesis of $\mathbf{1}$, we employed a heterologous expression approach using $A$. nidulans L08030. ${ }^{28}$ As $A$. nidulans LO8030 has a minimal metabolic background, it would facilitate downstream compound isolation compared to the complex metabolic profile of $A$. burnettii. We used multimarker AMA1-based fungal vectors (PYFAC) for expression, which facilitate combinatorial evaluation of gene cluster 

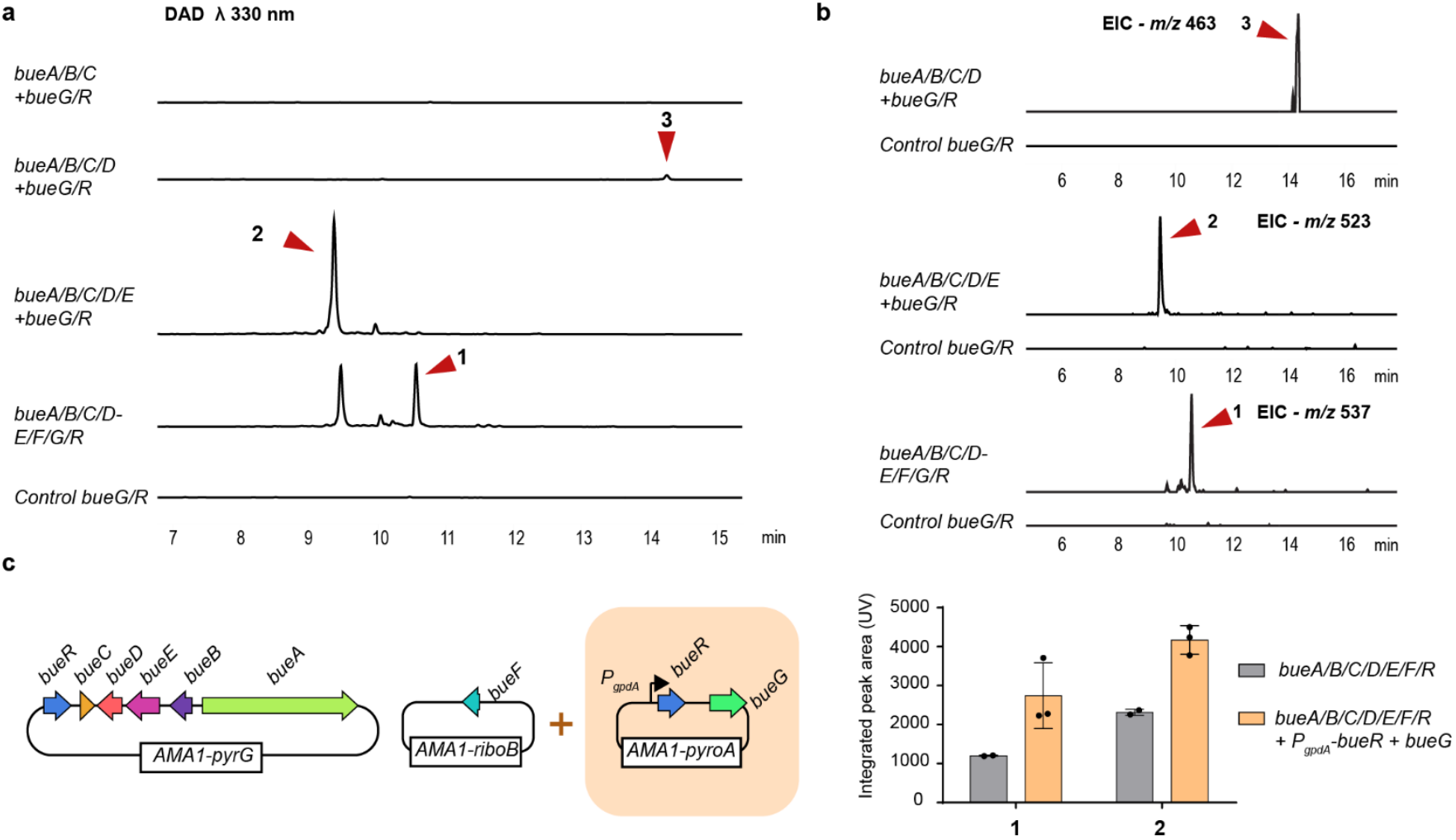

Figure 4 Analysis of metabolites encoded by the bue cluster following heterologous expression in A. nidulans. a. Representative HPLC traces ( 330 nm) of culture media extracts from strains expressing bue cluster genes. b. Extracted ion chromatograms (EICs) in negative ion mode showing production of burnettiene $A$ (1) and pathway intermediates. c. Strains harbouring the vector for bue $R$ transcription factor overexpression and the bue $G$ transporter produce higher compound titres. Values are the mean of biological replicates isolated from individual transformant colonies. Individual values are indicated as dots, and error bars represent standard deviations.

products. $^{29}$ As 1 is actively produced by $A$. burnettii MSTFP2249, ${ }^{4}$ we built vectors containing the bue genes under their native regulatory sequences. We built a pYFAC-pyrG vector containing an 18-kb region that encodes bue $A / B / C / D / E / R$, and a pYFAC-riboB vector containing bueF (Fig. 4C). In the related fusarielin BGC, overexpression of the TF FSL7 leads to an increased fusarielin titre in its native host. ${ }^{15}$ To evaluate if the titres of $\mathbf{1}$ could be increased by TF overexpression in a heterologous host context, we constructed a pYFAC-pyroA vector encoding the TF bueR under the strong constitutive promoter gpdA $\left(P_{g p d A}\right)$, and additionally included the transporter gene bueG (Fig. 4c).

LC-DAD-MS analysis of media extracts from the transformant $A$. nidulans strains expressing all the putative bue cluster genes (bueA/B/C/D/E/F/G/R) showed a peak matching 1 with $\mathrm{m} / \mathrm{z}$ of $537[\mathrm{M}-\mathrm{H}]^{-}$, which was not observed in the control strain (Fig. $4 a, b)$. The UV-vis spectrum matched the profile for $\mathbf{1}$ previously observed in A. burnettii extracts (Fig. S29+). When comparing strains with and without the vector for TF overexpression, we observed a $\sim 2$-fold increase in compound production in the strains containing the vector encoding $P_{\text {gpdA }}$-bue $R$ and bue (Fig. 4c). We decided to proceed with strains with TF overexpression to facilitate downstream compound isolation. We isolated 1 $(1.1 \mathrm{~g})$ from a large-scale solid-phase cultivation of $A$. nidulans (bue $A / B / C / D / E / F / G / R$ ) on jasmine rice. The NMR data for the isolated compound were identical to those for $\mathbf{1}$ isolated from
A. burnettii (Figs. S9-10†). This validates that the putative bue cluster is indeed responsible for the biosynthesis of 1 .

To elucidate the intermediates in the biosynthetic pathway to 1, we evaluated truncated versions of the bue cluster by heterologous expression in $A$. nidulans (Fig. S28+). We first hypothesised that the methyltransferase bueF would be responsible for the last step of biosynthesis. When the vector encoding the methyltransferase bueF was not transformed, we observed high production of compound 2 with a UV-vis spectrum matching to 1 and a mass ion $m / z$ of $523[\mathrm{M}-\mathrm{H}]^{-}$ (Figs. 3 and S29t). The difference in mass of $14 \mathrm{Da}$ was attributed to the presence of a free carboxylic acid in $\mathbf{2}$ compared to the methyl ester in $\mathbf{1}$. We then cloned a truncated version of the cluster also omitting the gene encoding the cytochrome p450 BueE. In the strains expressing bue $A / B / C / D / G / R$, we observed peak 3 at high retention time (14.24 $\mathrm{min}$ ) with a UV-vis spectrum similar to 1 and $\mathrm{m} / \mathrm{z} 463$ [M $-]^{-}$(Fig. 3 and S29+).

Next, we evaluated a truncated version of the cluster containing HR-PKS bueA, the ER bue $B$, and the $\alpha / \beta$ hydrolase bue $C$ to observe the first possible pathway intermediate. However, we did not observe any new peaks in the transformant $A$. nidulans strains expressing bue $A / B / C / G / R$ compared to the control (Fig. $3)$. For troubleshooting, we verified the correct sequence of vector pYFAC-bue $A / B / C / R$ by next generation sequencing (Fig. $\left.\mathrm{S} 30^{+}\right)$. In the homologous fusarielin cluster, deletion of the 


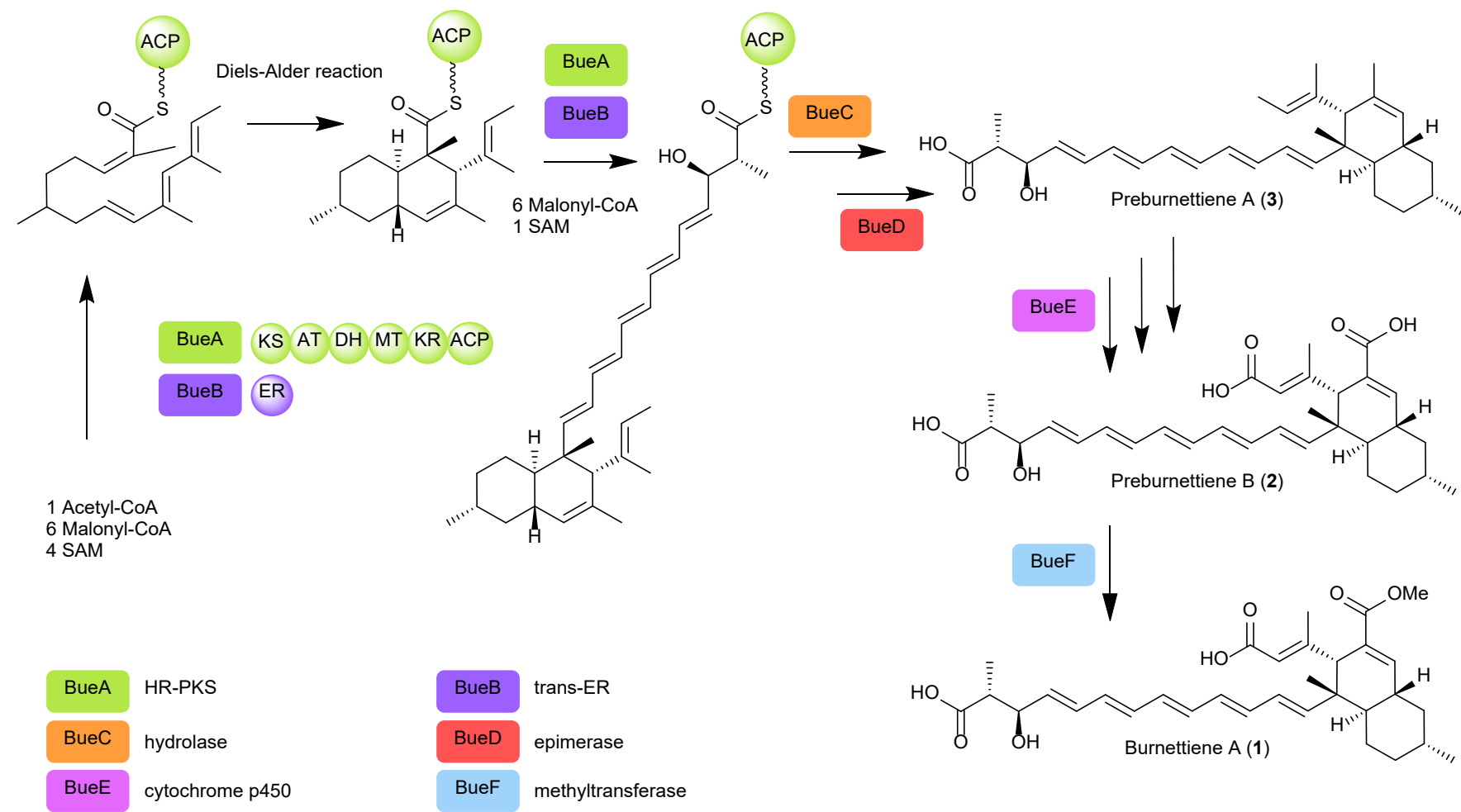

Figure 5 Proposed biosynthesis of burnettiene A (1). KS, ketosynthase; AT, malonyl-CoA acyl transferase; DH, dehydratase; MT, methyltransferase; $K R$, ketoreductase; ACP, acyl carrier protein; ER, enoyl reductase; SAM, S-adenosylmethionine.

epimerase gene results in the production of an unstable compound. ${ }^{16}$ Instability of the released product could also explain the lack of accumulation of the first bue cluster intermediate.

To elucidate the structures of $\mathbf{2}$ and 3, $1500 \mathrm{mg}$ and $200 \mathrm{mg}$ respectively were purified from large-scale cultivations on jasmine rice of the strains expressing bue $A / B / C / D / E / G / R$ or bue $A / B / C / D / G / R$ (Figs. S31-33+). HRESI(-)MS analysis of 2 revealed a deprotonated molecule $[\mathrm{M}-\mathrm{H}]^{-} \mathrm{m} / \mathrm{z} 523.2714$ indicative of a molecular formula $\mathrm{C}_{31} \mathrm{H}_{40} \mathrm{O}_{7} \quad(\Delta \mathrm{mmu}$ 1.3) containing one carbon and two hydrogen atoms fewer than 1 (Fig. S19+). The ${ }^{1} \mathrm{H}$ and ${ }^{13} \mathrm{C}$ NMR data for $\mathbf{2}$ were very similar to those for 1 (Figs. S12 and S13 and Table S2+), with the only significant difference being the absence of resonances associated with the methoxy group of the $5-\mathrm{CO}_{2} \mathrm{Me}$ ester $\left(\delta_{\mathrm{H}}\right.$ $\left.3.59, \mathrm{~s} ; \delta_{\mathrm{C}} 51.7\right)$. Detailed NMR analysis confirmed the structure as $\mathbf{2}$, herein named preburnettiene $\mathrm{B}$, as containing a free carboxylic acid group $\left(5-\mathrm{CO}_{2} \mathrm{H}\right)$ in place of the methyl ester in $\mathbf{1}$ (Fig. 5, Table S3, Figs. S14-S17+). Additionally, we observed that after 14 days of large-scale cultivation, 2 was completely converted to 1 (Fig, S31a†). Overall, these results demonstrate that BueF is responsible for methylation of the $5-\mathrm{CO}_{2} \mathrm{H}$ group on 2 and is required for the biosynthesis of $\mathbf{1}$ (Fig. 5). Methyltransferases that form a methyl ester are quite rare, as they usually form methoxy groups in polyketides. ${ }^{30}$

HRESI(-)MS analysis of 3 revealed a deprotonated molecule [M $-\mathrm{H}]^{-} \mathrm{m} / \mathrm{z} 463.3239$ indicative of a molecular formula $\mathrm{C}_{31} \mathrm{H}_{44} \mathrm{O}_{3}$ $(\Delta \mathrm{mmu} 2.1)$ containing four oxygen atoms fewer and four hydrogen atoms greater than 2 (Fig. S27+). The ${ }^{1} \mathrm{H}$ and ${ }^{13} \mathrm{C} N M R$ data for $\mathbf{3}$ were very similar to those for $\mathbf{2}$ (Figs. S20 and S21 and Table $\mathrm{S}^{+}$), with the only significant difference being the absence of signals for the carboxylic acid groups at C-1 and on $\mathrm{C}-5$, and the presence of signals attributable to two additional methyl groups $\left(\delta_{\mathrm{H}} 1.57, \mathrm{~d} ; \delta_{\mathrm{C}} 13.6\right.$ and $\left.\delta_{\mathrm{H}} 1.46, \mathrm{~s} ; \delta_{\mathrm{C}} 22.5\right)$. A COSY correlation between $\delta_{\mathrm{H}} 1.57$ and $\mathrm{H}-2$ confirmed the first methyl group to be at $\mathrm{C}-1$, while $\mathrm{HMBC}$ correlations from $\delta_{\mathrm{H}}$ 1.46 to $\mathrm{C}-4, \mathrm{C}-5$ and $\mathrm{C}-6$ confirmed the second methyl group to be on C-5 (Figs. S22-24t). Thus, the structure of intermediate 3 was assigned as shown in Fig. 5. This compound is herein named preburnettiene $\mathrm{A}$. We propose that the cytochrome $\mathrm{p} 450 \mathrm{BueE}$ catalyses regiospecific multi-oxidations at C-1 and 5-Me on 3 to the dicarboxylic acids on $\mathbf{2}$ (Fig. 5). Thus, the activity of bueE targeting the branched chains differs to FSL4 from the fusarielin BGC which is responsible of oxidising the decalin core. The triple oxidation of a methyl group to produce a carboxylic acid has been previously observed to be catalysed by a single cytochrome P450 enzyme in fungi. ${ }^{31,32}$ However, BueE has the particularity of catalysing the reaction in two terminal methyl groups.

Other smaller peaks with similar UV to 1 were observed more evidently in the scaled-up cultures (Fig. S31+). Among them, $\mathbf{4}$ is a non-polar burnettiene of a molecular weight of 462 and with a polyene UV with a bathochromic shift of $\sim 20 \mathrm{~nm}$ compared to the other burnettienes (Fig. S31b, $\mathrm{C}^{\dagger}$ ). However, 4 was prone to degradation and the quantity isolated was insufficient for structure elucidation.

To further investigate the absolute configurations of C-24 and C-25 in 1-3, we attempted to crystallise $\mathbf{1}$ and 2, which was not 
successful, perhaps due to the flexibility of the polyene moiety. However, in polyketides, the chirality of $\beta$-hydroxy group and $\alpha$ substituent can be predicted by analysis of the conserved residues on the KR domain. ${ }^{33}$ We investigated the KR domain of BueA by multiple sequence analysis to identify conserved fingerprint residues (Fig. S35t). We observed that BueA contained the signature leucine and aspartic acid from the LDD motif of B-type $\beta$-ketoreductases, which yields an $R$-hydroxy group, and a glutamine characteristic of B1-type KRs, which yield an $R$ - $\alpha$-substituent configuration (Fig. S35t). ${ }^{33,34}$ These results suggest that $\mathbf{1 - 3}$ likely possess $24 R, 25 R$ configurations, similar to fusarielin. ${ }^{35}$ However, exceptions to the rule have been observed, as in the case of the PKS Hpm8, ${ }^{36}$ so these stereochemical assignments are only tentative at this stage.

Equipped with 1-4, we evaluated their antibacterial, antifungal, antiparasitic, herbicidal and cytotoxic activities (Table 1 and Table $\left.\mathrm{S}^{+}\right)$. Compound $\mathbf{1}$ showed considerable cytotoxic activity against murine myeloma NS-1 cells (MIC $0.8 \mu \mathrm{g} / \mathrm{mL}$ ). Additionally, 1 showed early fungistatic activity against Candida albicans at $24 \mathrm{~h}$ cells (MIC $12.5 \mu \mathrm{g} / \mathrm{mL}$ ), although at $48 \mathrm{~h}$ growth was restored. Compound $\mathbf{4}$ showed activity against Bacillus subtilis (MIC $6.3 \mu \mathrm{g} / \mathrm{mL}$ ), while the activity of 1-3 against $B$. subtilis was limited at $48 \mathrm{~h}$.

Overall, our results demonstrate that the biosynthesis of $\mathbf{1}$ is encoded by the BGC composed of bueA/B/C/D/E/F/G/R. Considering the heterologous expression results, we propose the biosynthetic pathway to 1 outlined in Fig. 5. BueA, with the assistance of the trans-ER BueB, synthesises the backbone polyketide from malonyl-CoA and acetyl-CoA, with methylations derived from $S$-adenosylmethionine (SAM). A Diels-Alder reaction catalysed by BueA takes place at the heptaketide stage to form the trans-decalin ring from the acyclic substrate, in a mechanism resembling the cyclisation by LovB in lovastatin biosynthesis. However, LovB contains a condensation domain that plays a role in controlling the cyclisation, which is not present in BueA. ${ }^{24}$ Considering that the molecular basis of Diels-Alder formation within HR-PKS are still unknown, BueA serves as another example of PKS domain architecture that can perform decalin cyclisations.

After the decalin ring is formed, the chain extension proceeds until the polyketide product is released by the $\alpha / \beta$ hydrolase BueC. Then, the epimerase BueD is needed for the conversion to the first stable intermediate, 3. The cytochrome p450 BueE catalyses the oxidation of the methyl groups $\mathrm{C}-1$ and $5-\mathrm{Me}$ in $\mathbf{3}$ to carboxylic acid in $\mathbf{2}$. The $5-\mathrm{CO}_{2} \mathrm{H}$ group is then methylated by BueF to produce 1. The TF BueR and the transporter BueG are likely necessary for the expression and transport of the produced compounds, as they favour higher titres in the context of heterologous expression.
Table 1 In vitro biological activities of compounds 1-4

\begin{tabular}{cccccccc}
\hline & \multicolumn{8}{c}{ Minimum Inhibitory Concentration $(\mu \mathrm{g} / \mathrm{mL})^{\mathrm{a}}$} \\
\cline { 2 - 7 } Compound & \multicolumn{3}{c}{ Bs } & \multicolumn{2}{c}{ Ca } & \multicolumn{2}{c}{ NS-1 } \\
\cline { 2 - 7 } & 24 & 50 & 12.5 & $>200$ & 0.8 & 0.8 \\
\hline $\mathbf{1}$ & 50 & 100 & $>200$ & $>200$ & 50 & 50 \\
3 & 12.5 & 50 & $>200$ & $>200$ & 25 & 25 \\
4 & 6.3 & 6.3 & $>200$ & $>200$ & 50 & 50 \\
\hline
\end{tabular}

a Bs, Bacillus subtilis (ATCC 6633); Ca, Candida albicans (ATCC 10231); NS-1, Murine myeloma NS-1 (ATCC TIB-18).

\section{Conclusions}

In conclusion, we have characterised the structure and biosynthesis of burnettiene A (1) from $A$. burnettii, which has been shown to be a biosynthetically talented fungus harbouring a large number of BGCs. ${ }^{4,6}$ Interestingly, 1 contains one of the longest polyketide chain (tridecaketide) known to form decalin structures. ${ }^{11} A$. burnettii also produces the unusually long tetradecaketide burnettramic acids. ${ }^{6}$ Compared to fusarielin A (Fig. 1), where the decalin core is highly oxidised, $\mathbf{1}$ has the branched chains highly oxidised with a methyl ester and carboxylic acid moieties. We have also investigated the biosynthesis of 1 by evaluating truncated gene cluster variants. This led to the isolation and characterisation of the intermediates preburnettienes A and B (3 and 2). We also identified the functions of the tailoring enzymes BueF and BueE. Considering the relevant bioactivities of related decalin structures, these enzymes have the potential to be used for the biocombinatorial decoration of related scaffolds to create novel derivatives. ${ }^{37}$ Additionally, carboxyl methyltransferases such as BueF are of biotechnological interest. ${ }^{38}$

Heterologous expression of bue genes in the chassis $A$. nidulans proved a more favourable strategy for the production and isolation of 1 than in the native host, obtaining a $\sim 45$-fold increase in the yield of $\mathbf{1}$ per $\mathrm{kg}$ of substrate compared to $A$. burnettii. ${ }^{4}$ Interestingly, we also observed that the strains of $A$. nidulans harbouring AMA1-based episomal vectors, which require auxotrophy selection for maintenance, can sustain production in grain substrates such as jasmine rice with yields as high as $468 \mathrm{mg} / \mathrm{kg}$ in the case of $\mathbf{2}$. Additionally, we showed that TF overexpression can be effective to upregulate AMA1encoded heterologous BGCs in A. nidulans, a strategy also used by $\mathrm{Hu}$ et al. ${ }^{29}$ Overall, this work highlights the benefits of heterologous expression in an engineered $A$. nidulans chassis even for BGCs that are active on their native host. ${ }^{20}$

Finally, this work contributed to the understanding of the widely spread burnettiene and fusarielin BGC family. As uncharacterised clusters in other Aspergilli, for example $A$. fumigatus cluster $4,2,27$ present a higher identity and synteny 
with bue gene cluster than to the FSL gene cluster, they are likely to encode compounds structurally more similar to burnettienes than fusarielins (Fig. S36t). Thus, the results from this work can help interpret this extensive and elusive cluster family in those fungi.

\section{Conflicts of interest}

There are no conflicts to declare.

\section{Acknowledgements}

We thank Berl Oakley for A. nidulans L08030 strain and members of Lister Lab (UWA), specifically Jahnvi Pflüger, for whole plasmid sequencing. This research was funded, in part, by the Australian Research Council (FT160100233, FT130100142) and the Cooperative Research Centres Projects scheme (CRCPFIVE000119). HRMS data were acquired by the Australian Proteome Analysis Facility, supported under the Australian Government's National Collaborative Research Infrastructure Strategy (NCRIS).

\section{References}

J. Romsdahl and C. C. C. Wang, Medchemcomm, 2019, 10, 840-866. N. P. Keller, Nat. Rev. Microbiol., 2019, 17, 167-180. H. Li, A. E. Lacey, S. Shu, J. A. Kalaitzis, D. Vuong, A. Crombie, J. Hu, C. L. M. Gilchrist, E. Lacey, A. M. Piggott and Y.-H. Chooi, Org. Biomol. Chem., 2021, 19, 587-595. C. L. M. Gilchrist, H. J. Lacey, D. Vuong, J. I. Pitt, L. Lange, E. Lacey, B. Pilgaard, Y.-H. Chooi and A. M. Piggott, Fungal Genet. Biol., 2020, 143, 103435.

H. Li, C. L. M. Gilchrist, C. S. Phan, H. J. Lacey, D. Vuong, S. A. Moggach, E. Lacey, A. M. Piggott and Y.-H. Chooi, J. Am. Chem. Soc., 2020, 142, 7145-7152.

H. Li, C. L. M. Gilchrist, H. J. Lacey, A. Crombie, D. Vuong, J. I. Pitt, E. Lacey, Y.-H. Chooi and A. M. Piggott, Org. Lett., 2019, 21, 1287-1291.

H. J. Lacey, C. L. M. Gilchrist, A. Crombie, J. A. Kalaitzis, D. Vuong, P. J. Rutledge, P. Turner, J. I. Pitt, E. Lacey, Y.-H. Chooi and A. M. Piggott, Beilstein J. Org. Chem., 2019, 15, 2631-2643.

N. K. Chaudhary, J. I. Pitt, E. Lacey, A. Crombie, D. Vuong, A M. Piggott and P. Karuso, J. Nat. Prod., 2018, 81, 15171526.

J. I. Pitt, L. Lange, A. E. Lacey, D. Vuong, D. J. Midgley, P. Greenfield, M. I. Bradbury, E. Lacey, P. K. Busk, B. Pilgaard, Y.-H. Chooi and A. M. Piggott, PLoS One, 2017, 12, e0170254.

0 H. J. Lacey, D. Vuong, J. I. Pitt, E. Lacey and A. M. Piggott, Aust. J. Chem., 2016, 69, 152-160.

G. Li, S. Kusari and M. Spiteller, Nat. Prod. Rep., 2014, 31, 1175-1201.

J. W. Sims, J. P. Fillmore, D. D. Warner and E. W. Schmidt, Chem. Commun., 2005, 186-188. W. Xu, Y. H. Chooi, J. W. Choi, S. Li, J. C. Vederas, N. A. Da

Silva and Y. Tang, Angew. Chemie - Int. Ed., 2013, 52, 64726475.

\section{0, 37, 1568-1588.}

J. L. Sørensen, F. T. Hansen, T. E. Sondergaard, D. Staerk, T. V. Lee, R. Wimmer, L. G. Klitgaard, S. Purup, H. Giese and R. J. N. Frandsen, Environ. Microbiol., 2012, 14, 1159-1170. A. Droce, W. Saei, S. H. Jørgensen, R. Wimmer, H. Giese, R. D. Wollenberg, T. E. Sondergaard and J. L. Sørensen, Molecules, 2016, 21, 1710.

H. Fujimoto, H. Aoyama, T. Noguchi-Yachide, Y. Hashimoto and H. Kobayashi, Chem. Pharm. Bull., 2008, 56, 298-304. T. Ugai, A. Minami, R. Fujii, M. Tanaka, H. Oguri, K. Gomi and H. Oikawa, Chem. Commun., 2015, 51, 1878-1881. B. Perlatti, G. Harris, C. B. Nichols, D. I. Ekanayake, J. A. Alspaugh, J. B. Gloer and G. F. Bills, J. Nat. Prod., 2020, 83, 2718-2726.

L. K. Caesar, N. L. Kelleher and N. P. Keller, Fungal Genet. Biol., 2020, 144, 103477.

Y. H. Chooi and Y. Tang, J. Org. Chem., 2012, 77, $9933-$ 9953.

B. D. Ames, C. Nguyen, J. Bruegger, P. Smith, W. Xu, S. Ma, E. Wong and S. Wong, Proc. Natl. Acad. Sci., 2012, 109, 11144-11149.

L. Li, P. Yu, M. Tang, Y. Zou, S. Gao, Y. Hung, M. Zhao, K. Watanabe, K. N. Houk and Y. Tang, J. Am. Chem. Soc., 2016, 138, 15837-15840.

S. M. Ma, J. W. H. Li, J. W. Choi, H. Zhou, K. K. M. Lee, V. A. Moorthie, X. Xie, J. T. Kealey, N. A. Da Silva, J. C. Vederas and Y. Tang, Science, 2009, 326, 589-592. H.-N. Lyu, H.-W. Liu, N. P. Keller and W.-B. Yin, Nat. Prod. Rep., 2019, 37, 6-16.

C. L. M. Gilchrist, T. J. Booth, B. van Wersch, L. van Grieken, M. H. Medema and Y.-H. Chooi, Bioinforma. Adv., vbab016. A. L. Lind, J. H. Wisecaver, C. Lameiras, P. Wiemann, J. M. Palmer, N. P. Keller, F. Rodrigues, G. H. Goldman and A. Rokas, PLoS Biol., 2017, 15, e2003583.

Y. M. Chiang, M. Ahuja, C. E. Oakley, R. Entwistle, A. Asokan, C. Zutz, C. C. C. Wang and B. R. Oakley, Angew. Chemie - Int. Ed., 2016, 55, 1662-1665.

J. Hu, F. Sarrami, H. Li, G. Zhang, K. A. Stubbs, E. Lacey, S. G. Stewart, A. Karton, A. M. M. Piggott and Y.-H. Chooi, Chem. Sci., 2019, 10, 1457-1465.

Q. Yang, W. Wang, Y. Lin, Y. Lin, Z. Tang, J. Wang, J. Tao, W. Tang and W. Liu, Org. Biomol. Chem., 19, 6638-6643. H. Li, J. Hu, H. Wei, P. S. Solomon, D. Vuong, E. Lacey, K. A. Stubbs, A. M. Piggott and Y.-H. Chooi, Org. Lett., 2018, 20, 6148-6152.

K. C. Van De Bittner, M. J. Nicholson, L. Y. Bustamante, S. A. Kessans, A. Ram, C. J. Van Dolleweerd, B. Scott and E. J. Parker, J. Am. Chem. Soc., 2018, 140, 582-585.

A. T. Keatinge-Clay, Chem. Biol., 2007, 14, 898-908. C. B. Bailey, M. E. Pasman and A. T. Keatinge-Clay, Chem. Commun., 2016, 52, 792-795.

K. Furihata, R. Sunaga, N. Morisaki and S. Iwasaki, J. Antibiot. (Tokyo)., 1995, 48, 42-52.

H. Zhou, Z. Gao, K. Qiao, J. Wang, J. C. Vederas and Y. Tang, Nat. Chem. Biol., 2012, 8, 331-333. 

Chem. Biol., 2020, 58, 45-53.

38 L. C. Ward, H. V. McCue and A. J. Carnell, ChemCatChem, 